\title{
Pulmonary involvement in diffuse cutaneous systemic sclerosis: broncheoalveolar fluid granulocytosis predicts progression of fibrosing alveolitis
}

\author{
Christian Witt, Adrian C Borges, Mathias John, Ingo Fietze, Gert Baumann, \\ Andreas Krause
}

\begin{abstract}
Objective-The clinical course of fibrosing alveolitis (FA) in patients with systemic sclerosis (SSc) may vary considerably from stable condition for years to continuous fatal progression. This prospective study aimed at identifying the prognostic value of bronchoalveolar lavage fluid (BALF) analysis in FASSc. Methods-Seventy three consecutive patients with SSc and clinical signs of pulmonary involvement were enrolled. Every patient underwent clinical examination, lung function tests, computed tomography (CT), gallium scan, echocardiography, and bronchoalveolar lavage (BAL). Forty nine patients, 26 with pathological and 23 with normal BALF findings were prospectively followed up for two years and re-evaluated annually.
\end{abstract}

Results-At baseline, 51 subjects (70\%) showed radiological signs of lung fibrosis and/or alveolitis by CT and diffusion capacity for carbon monoxide (DLco) was decreased in 47 patients $(64 \%)$. Thirty five patients $(48 \%)$ had pathological BALF findings. BALF differential counts included BALF granulocytosis in 18, BALF lymphocytosis in 12 , and a mixed increase of both granulocytes and lymphocytes in five patients. On follow up, a progression of FA with a significant decrease of DLco was only observed in patients with BALF granulocytosis. In contrast, patients with BALF lymphocytosis or normal BALF cell count had stable lung funtion parameters during the study period. In none of our patients echocardiography showed evidence of pulmonary hypertension.

Conclusion-BALF granulocytosis predicts progression of FA with deterioration of lung function, which is most sensitively monitored by DLco. Immunosuppressive treatment is recommended in patients with granulocytic FASSc.

(Ann Rheum Dis 1999;58:635-640)

Systemic sclerosis (SSc) is a connective tissue disease that affects the skin and internal organs. The pathogenesis is characterised by autoimmune processes of unknown aetiology that eventually lead to fibrotic vasculopathy and diffuse accumulation of extracellular matrix proteins. About $70 \%$ of patients with
SSc develop pulmonary involvement with inflammation and fibrosis of the interstitium, the alveoli and peribronchial tissue. Vasculopathy may lead to pulmonary hypertension even in the absence of significant fibrosis. ${ }^{1-3}$

Pulmonary complications are the leading cause of mortality in SSc. Early diagnosis and immunosuppressive treatment of fibrosing alveolitis (FA) may therefore improve the prognosis of SSc patients. ${ }^{4}$ However, recognition of interstitial lung manifestation in SSc may be delayed because the clinical signs are often mild and insidious. Moreover, the disease course of FA associated with SSc (FASSc) may vary considerably making treatment decisions difficult. In some patients lung function may remain stable for years even in later disease stages, while others may experience fatal progressive FASSc. ${ }^{5}$ To minimise the risk of invasive over-diagnosis and over-treatment parameters to assess the disease extent and to predict the prognosis of FASSc are needed.

In previous papers by us and others, late inspiratory crackles, increased 67-gallium uptake, and abnormal broncheoalveolar lavage fluid (BALF) findings have been shown to be the most valuable methods to diagnose alveolitis in collagen vascular diseases including SSc, and impaired diffusing capacity for carbon monoxide (DLco), BAL neutrophilia, and interstitial fibrosis documented by computed tomography (CT) best reflected the extent of FASSc. ${ }^{78}$ However, only little is known about the prognostic relevance of findings obtained by these methods.

This study therefore aimed at evaluating if BALF analysis permits the determination of inflammatory activity and prediction of the prognosis of FASSc. The results showed that BALF granulocytosis predicted progressive FASSc. After a follow up period of 24 months only patients with BALF granulocytosis showed a deterioration of DLco. In contrast, patients with BALF lymphocytosis or normal BALF cell count had stable lung function parameters during the study period.

\section{Methods}

PATIENTS

Seventy three consecutive patients with diffuse SSc according to the American College of Rheumatology (formerly, the American Rheumatism Association) preliminary criteria for the diagnosis of SSc were enrolled in this study as described previously. ${ }^{79}$ All patients had been 
Table 1 Clinical characteristics, serological and BAL data, radiological and scintigraphic findings in SSc patients $(n=73)$

Clinical and demographic characteristics male/female

mean (SD) age

median duration of SSc (range)

cigarette smokers

past smokers

dyspnea

dry cough

crackles

Serological data

anti-nuclear antibodies

anti-Scl 70 antibodies

BAL data

pathological BAL

granulocytosis

lymphocytosis

combined granulocytosis/lymphocytosis

Radiological and scinigraphic findings

chest radiography: alveolitis/fibrosis

CT: alveolitis/fibrosis

increased 67 gallium uptake

$16 / 57$
$54.4(9.6)$
5.0 years $(2-17)$
$9(12 \%)$
$12(16 \%)$
$57(78 \%)$
$40(55 \%)$
$35(47 \%)$
$66(90 \%)$
$32(44 \%)$
$35(48 \%)$
$18(52 \%$ of pathological BAL)
$12(34 \%$ of pathological BAL)
$5(14 \%$ of pathological BAL $)$
$39(53 \%)$
$51(70 \%)$
$23(31 \%)$

referred from the departments of dermatology and rheumatology to our pneumological outpatient clinic because of clinical, lung functional, and/or CT signs of interstitial lung disease. Patients with limited cutaneous SSc or CREST syndrome were excluded from the study. Sixty six patients (90\%) were positive for antinuclear antibodies (ANA), and 32 (44\%) had antibodies against topoisomerase I (antiScl 70).

The mean (SD) age of the 57 women and 16 men was 54.4 (9.6) years (20-80 years). Nine patients were active cigarette smokers and 12 were past smokers (that is, had stopped smoking at least one year before study entry). The duration of SSc ranged from 2 to 17 years (median 5.0 years), as determined from the time of diagnosis to entry into this study. During the study 46 patients (63\%) were receiving a low dose prednisolone therapy $(<12.5$ $\mathrm{mg} /$ day), six patients received low dose prednisolone plus azathioprine (100-150 mg/day), and two patients each were receiving azathioprine or D-penicillamine $(450 \mathrm{mg})$ monotherapy. Seventeen patients $(23 \%)$ did not receive any immunosuppressive treatment. Table 1 summarises the demographic data. All patients gave their informed consent to participate in this study.

Forty nine patients, 26 of whom with pathological BALF findings and 23 with normal BALF results were prospectively followed up for two years and re-evaluated anually by clinical examination and lung function tests.

CLINICAL EXAMINATION

Patients were asked about dry cough, dyspnea at rest and at exertion, and smoking habits. Clinical examination was performed by two independent experienced physicians and was focused on auscultation for reproducible late inspiratory crackles (sclerophonia) at the lung basis and above the middle lobes.

RADIOLOGICAL INVESTIGATIONS

Standard posteroanterior and lateral chest radiographs were performed in upright position. Thoracic high resolution CT was performed in supine position and in maximal inspiration using a Somatom plus scanner in a high spatial frequency algorithm $(120 \mathrm{kV}, 255$ $\mathrm{mA}$, Siemens, Erlangen, Germany). Consecutive dynamic scans at $10 \mathrm{~mm}$ slice spacing with $1 \mathrm{~mm}$ slice thickness were obtained. In some patients with increased opacification in the posterobasal segments, an additional investigation in prone position was performed, to ensure that opacification was not caused by gravity dependent perfusion. ${ }^{10}$ The scans were reconstructed with a high resolution bone algorithm (window level -500 to $-600 \mathrm{HU}$; window width 1800-2000 HU). Two radiologists independently viewed the CT scans in a random order without knowledge of the patients' clinical condition. CT findings were categorised qualitatively as normal or abnormal, regardless of the specific appearence (that is, ground glass appearance, honeycombing, septal lines, disruption of the normally smooth pleural contour).

\section{LUNG FUNCTION TEST}

Spirometry and body plethysmography (Siregnost FD 40/FD 91, Siemens) were performed to determine standard dynamic and static ventilatory parameters including forced vital capacitiy (FVC), forced expiratory volume in 1 sec $\left(\mathrm{FEV}_{1}\right)$, total lung capacity (TLC), and residual volume (RV). The pulmonary diffusing capacity for carbon monoxide (DLco) and the specific pulmonal diffusing capacity (Kco) were determined by the single breath method (Transferscreen II, Fa. Jäger, Würzburg, Germany). Values for $\mathrm{FVC}, \mathrm{FEV}_{1}, \mathrm{FEV}_{1} / \mathrm{FVC}$, TLC, RV, RV/TLC, Kco, and DLco were expressed as percentage of the age, sex, and height related predictive normal values. Results below $80 \%$ of the respective normal value were considered pathological.

\section{7-GALLIUM SCINTIGRAPHY}

Scintigraphy was performed 48 hours after intravenous injection of $100 \mathrm{MBq} 67$-gallium citrate by large field gammacamera (Picker $4 / 15)$ in anterior and posterior position. Enrichment of 67-gallium in the lung parenchyma was semi-quantitatively determined, assessed in relation to the enrichment in the liver.

BRONCHOALVEOLAR LAVAGE (BAL)

BAL was performed as recommended by the American Thoracic Society and as described previously using an Olympus BF 1T20 fibreoptic bronchoscope. ${ }^{11}$ In brief, after premedication with atropine $(0.5 \mathrm{mg})$ intravenously, hydrocodon (15 mg) subcuteaneously, and diazepam (5-10 mg) intravenously, and local anaesthesia with prilocaine the bronchoscope was wedged into a segment bronchus of the the right middle lobe and $150 \mathrm{ml}$ of $0.9 \%$ natrium chloride solution $\left(37^{\circ} \mathrm{C}\right)$ were instilled in $20 \mathrm{ml}$ aliquots. Fluid was gently aspirated, filtered through gauze for elimination of mucus and pooled in sterile silicone tubes at $4^{\circ} \mathrm{C}$. After centrifugation $\left(4^{\circ} \mathrm{C}, 10 \mathrm{~min}\right.$ at $\left.1400 \mathrm{rpm}\right)$ the cell pellet was washed twice with phosphate buffered saline solution. BAL differential cell counts were performed on cytospin preparations stained by the May-Grünwald-Giemsa 
method. Six hundred cells each were differentiated microscopically (magnification $\times 1000$ ) on three different slides in random fields for macrophages, neutrophiles, lymphocytes and eosinophils. According to normal values obtained by the same BAL procedure in our laboratory to those reported in non-smoking control subjects by other investigators, ${ }^{12}{ }^{13}$ the following BAL differential cell counts were classified as pathological: in non-smokers $>15 \%$ lymphocytes, $>3 \%$ neutrophils, and/or $>0.5 \%$ eosinophils; in smokers $>7 \%$ lymphocytes, $>3 \%$ neutrophils, and $/$ or $>0.5 \%$ eosinophils. Pathological BAL cell counts were differentiated in lymphocytic, granulocytic (neutrophilia and/or eosinophilia) and mixed forms (combination of lymphocytosis and granulocytosis). Interpretation of BALF analysis was blinded to knowledge of clinical, radiological, and lung function parameters.

TRANSTHORACIC ECHOCARDIOGRAPHY

All patients underwent two dimensional transthoracic echocardiography at the time of enrolment and at two years follow up. Examinations were performed in the left lateral decubitus position on a Toshiba SSH 270 A ultrasound system (Tokyo, Japan) using a 3.75- and $2.5-\mathrm{MHz}$ transducer and recorded on VHS video. Parasternal long axis, mid-ventricular parasternal short axis, apical four chamber, and apical two chamber images were acquired. All echocardiograms were reviewed on the video tapes and a consensus was achieved by two observers unaware of the clinical data. Right ventricular dilatation, paradoxal movement of the interventricular septum, tricuspidal regurgitation were interpreted as indirect signs for pulmonary hypertension. Calculation of the peak pulmonary artery pressure was performed with the tricuspid regurgitant jet and the Bernoulli equation. ${ }^{14}$

\section{STATISTICS}

Results are expressed as means (SD) unless otherwise indicated. For between groups Student's $t$ test was used. Differences between baseline and follow up parameters within groups were analysed by the paired $t$ test. Correlations between nominally scaled parameters were tested by the $\chi^{2}$ test with Yates's correction and dependencies between metrically scaled parameters were evaluated using the Keandall and Pearson correlation coefficient (probability level 1\%). p Values less than 0.05 in one and multivariant data analyses (logistic regression) were considered to be statistically significant. Statistical analyses were performed using StatView II data processing software (version 1997).

\section{Results}

ASSESSMENT OF FA AT BASELINE

All 73 patients had clinical symptoms suggestive of pulmonary involvement of SSc including mild to severe dyspnea at exertion (78\%), dry cough $(55 \%)$, and late inspiratory crackles upon lung auscultation (47\%). In 51 subjects $(70 \%)$ radiological signs of lung fibrosis and/or alveolitis could be observed by CT. Pathologi- cal BALF findings indicative of active alveolitis were demonstrated in 35 patients (48\%). BALF differential counts included BALF granulocytosis in 18, BALF lymphocytosis in 12 , and a mixed increase of both granulocytes and lymphocytes in five patients. The nine smokers included in this study had less lymphocytes in BALF differential cell counts (except for three patients with BALF lymphocytosis), but otherwise did not differ in BALF analyses from past smokers or nonsmokers. Low dose prednisolone treatment was given to $83 \%$ of the patients with pathological BALF results and $71 \%$ of the patients with normal BALF (difference not significant) with a median dose of $10 \mathrm{mg} /$ day in both groups.

Comparison of BALF findings with the results of clinical examination and imaging techniques showed a strong correlation $(p<0.01)$ between late inspiratory crackles (audible in 35 of 73 patients) and increased BALF lymphocyte or granulocyte counts, respectively, thus demonstrating the high diagnostic value of thorough lung auscultation. Gallium scanning turned out to be less sensitive in detecting alveolitis in that only 23 subjects $(31 \%)$ had an increased pulmonary 67-gallium uptake. Interestingly, a pathological gallium scan was more frequently found in patients with BALF lymphocytosis than in patients with other BALF findings $(p<0.05)$. Only three of the patients with increased 67-gallium uptake had a normal BALF cell count, which shows the high specificity of a pathological gallium scan. CT showed an abnormal interstitial pattern in $89 \%$ of SSc patients with a pathological differential cell count in BALF. Only four (11\%) of the 35 patients with inflammatory BALF had unremarkable CT findings. However, 20 patients showed pathological CT findings but normal BALF results.

Serological studies showed that BALF granulocytosis was correlated with the presence of anti-Scl 70 autoantibodies. Patients with anti-Scl 70 antibodies significantly more often had pathological BALF findings (68\%) than patients who were anti-Scl 70 negative $(34 \%, \mathrm{p}<0.05)$. Of the patients with inflammatory BALF, $73 \%$ with BALF granulocytosis were anti-Scl 70 positive, while only $42 \%$ with BALF lymphocytosis showed this autoantibody $(\mathrm{p}<0.05)$.

The lung function test that most sensitively detected pulmonary impairment was DLco, which was decreased in 47 patients $(64 \%), 38$ $(81 \%)$ of whom had a pathological CT. In contrast, FVC and TLC were reduced in only 38 $(52 \%)$ and 15 patients $(21 \%)$, respectively. Of special interest, patients with BALF granulocytosis had a significantly reduced DLco (66 (18) $\%$ of predicted value) as compared with patients with a lymphocytic alveolitis (87 (13) $\%, p<0.01$ ) or a normal BALF cell count (79 (17)\%, p<0.05, fig 1). In all 73 patients echocardiography was unremarkable and without evidence for right ventricular dysfunction or pulmonary hypertension. 

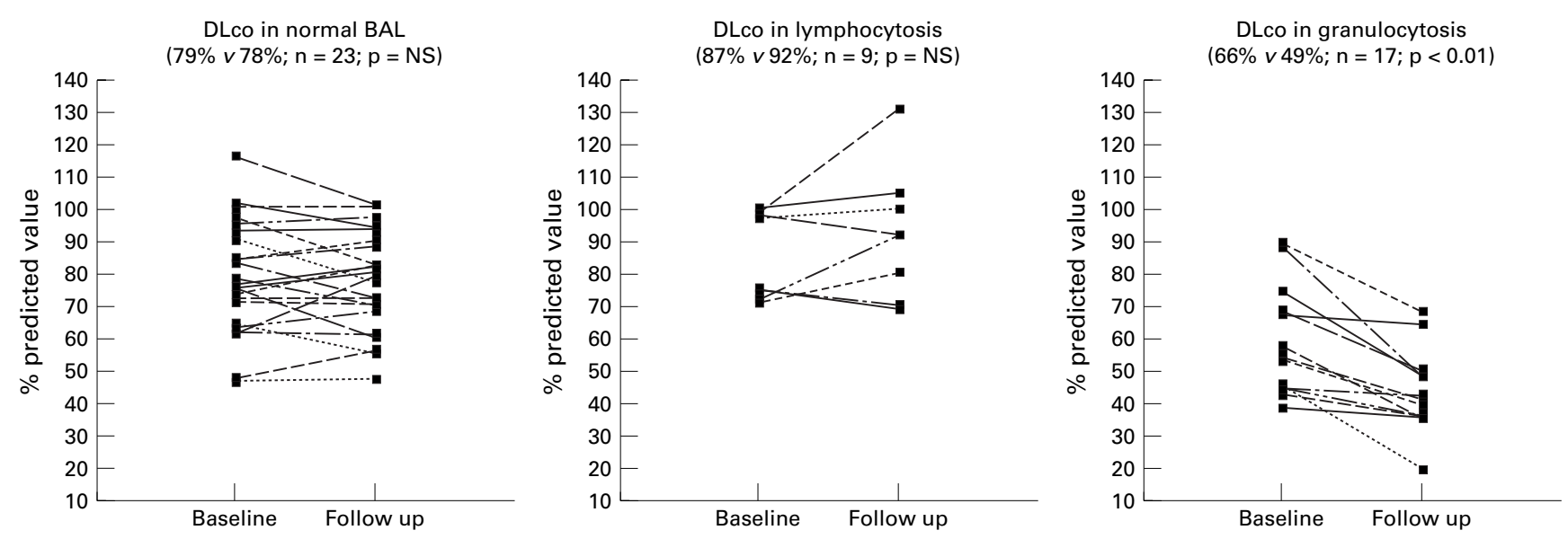

Figure 1 DLco of SSc patients with normal BALF analysis, BALF lymphocytosis, and BALF granulocytosis, respectively, at baseline and after two years of follow up.

FOLLOW UP STUDY

Forty nine patients (seven male, 42 female; mean (SD) age 51.4 (9.1) years), 26 with pathological and 23 with normal BALF findings, were prospectively followed up for two years and re-evaluated annually by clinical examination, and lung function tests. Five patients with mixed BALF granulocytosis/ lymphocytosis and four smokers with normal BALF were excluded from follow up as these patient groups were too small for evaluation. The remaining four patients with pathological BALF and 11 patients with normal BALF were lost to follow up because of the changes in the health care system in Germany after the reunionification.

All but three patients received a low dose prednisolone treatment. Two patients with BALF granulocytosis additionally took azathioprine. Patients with normal BALF cell counts at baseline had stable lung function parameters except for two patients who had a significant decrease of DLco during the study period. Of the patients with abnormal BALF differential cell counts, those nine patients with BALF lymphocytosis also remained stable with regard to lung function. In contrast, the 17 patients with BALF granulocytosis showed a signifcant decrease of DLco from 65 (18)\% of the predicted value at baseline to 49 (15)\% after two years $(\mathrm{p}<0.01)$ (fig 1$)$. However, TLC $(86 \%(17) v 84(20) \%)$ and FVC (72 (18) v 70 $(20) \%)$ remained unchanged. Of special interest, none of the 49 patients had developed any clinical or echocardiographic signs of pulmonary hypertension during the two year follow up period.

\section{Discussion}

The majority of patients with SSc develop pulmonary manifestations. Patients with diffuse scleroderma and anti-topoisomerase I autoantibodies especially are at high risk for FASSc. ${ }^{13} 15$ However, the clinical course of FASSc may vary considerably. While some patients have stable lung function parameters for years others may develop incapacitating or even fatal pulmonary fibrosis.

Though the overall therapeutic options in SSc are poor, there is evidence that corticosteroids and immunosuppressive treatment may significantly slow the progression of FASSc. ${ }^{16}$ Inhibition of local cytokine production may contribute to the effect of this treatment. ${ }^{17}$ However, there are only few prognostic markers that allow the prediction of the progression of pulmonary fibrosis and that may guide therapeutic decisions in FASSc.

Previous studies have shown that in SSc BALF neutrophilia is associated with extensive fibrotic disease as indicated by CT findings ${ }^{18}$ and DLco is the lung function parameter that best reflects the extent of FASSc. $^{8}$ The prognostic significance of these findings has not been evaluated in these studies. Recently, Behr et $a l^{16}$ reported that untreated FASSc patients with abnormal BAL showed a deterioration of their lung function after a median follow up period of of 57 weeks in contrast with treated patients or patients with normal BAL who remained stable. However, the prognostic significance of BALF differential cell counts was not investigated. Jacobsen $e t a l^{15}$ found in their retrospective study of patients with SSc that the presence of anti-Scl 70 antibodies was associated with a restrictive ventilatory pattern, and that isolated progression of DLco reduction was related to anti-centromere antibodies. They concluded that two different types of pulmonary damage exist with different pathogenesis. No BALF data were available in this study to further support this hypothesis.

The present prospective investigation focused on evaluating BALF analysis as a prognostic parameter for FASSc. Evaluation at baseline showed that late inspiratory crackles are a sensitive clinical parameter for alveolitis that correlated to pathological BALF differential cell counts. Impairement of lung function was best identified by a decreased DLco. This finding is in line with the results reported by Wells et al. ${ }^{8}$ Reduction of DLco was related to BALF granulocytosis in that patients with this BALF result had a significantly lower DLco than patients with normal BALF or BALF lymphocytosis. Anti-Scl 70 seropositivity indicated an increased incidence of FASSc and BALF granulocytosis. These findings therefore corroborate earlier reports that patients with anti-Scl 70 antibodies are more likely to develop FASSc than seronegative patients. ${ }^{13} 15$ CT was pathological in $89 \%$ of patients with 
increased BALF cell counts-that is, active alveolitis-but also showed interstitial changes in $53 \%$ of patients with normal BALF findings - that is, FASSc that was not active at the time of investigation. Thus, CT is sensitive in detecting FASSc, but cannot substitute for BALF in completely assessing the inflammatory activity of pulmonary involvement in SSc. Scintigraphy with 67 -gallium did not provide further information and seems to be unnecessary in assessing FASSc.

Of the 49 FASSc patients who were followed up for two years only those with BALF granulocytosis at baseline showed a significant disease progression with a marked reduction of DLco while almost all patients with normal BALF findings or BALF lymphocytosis had stable lung function parameters during the study period. Only two patients in the normal BALF group showed a significant decrease of DLco. It may be hypothesised that these patients had had an intermittent disease flare with (granulocytic?) alveolitis that could not be detected at the follow up visits, but had lead to a deterioration of lung function. The results demonstrate that BALF granulocytosis is an important prognostic parameter in FASSc that indicates a progressive inflammatory process eventually leading to a deterioration of lung function. In contrast, BALF lymphocytosis rather reflects a more benign course of FASSc.

Our data confirm and extend earlier studies on the prognostic relevance of BALF in FASSc. ${ }^{4}{ }^{16}$ As we did not perform serial BAL examinations within this study it cannot be answered whether granulocytic and lymphocytic alveolitis reflect two distinct subtypes of FASSc or just different stages in the inflammatory process. Many of our patients with BALF lymphocytosis and even some with normal BALF had pathological interstitial pattern by CT and decreased lung function parameters. This makes it probable that BALF granulocytosis indicates an active state of FASSc while BALF lymphocytosis and even normal BALF may be found in inactive phases. This would mean that FASSc is not necessarily a chronically progressive disease, but may take an undulating course with flares and remissions as has been observed in most rheumatic disorders.

Besides alveolitis pulmonary hypertension is a prognostically important manifestation of SSc. Echocardiography, a sensitive and noninvasive method to diagnose pulmonary hypertension, was performed in all patients studied at baseline and after two years. In none of our patients clinical or echocardiographic evidence of pulmonary hypertension was observed. Although slight increases of the pulmonary artery pressure might have been missed by echocardiography, clinically relevant pulmonary hypertension as a possible explanation for the reduction of DLco observed could be ruled out. This is quite unusual in light of the high prevalence rates of pulmonary hypertension given in the literature. ${ }^{19}$ Possible explanations include the relatively short disease duration of many of our patients and the exclusion of patients with CREST syndrome, who are at a much higher risk for developing pulmonary hypertension than patients with diffuse Ssc. ${ }^{19}$

Progressive FASSc has many features in common with FA of unknown origin (cryptogenic FA) including histology, though cryptogenic FA usually has a much worse overall prognosis than FASSc. The typical finding in cryptogenic FA is a pronounced BALF granulocytosis. ${ }^{68}$ Only recently, Salaffi et $a l^{20}$ reported that in patients with pulmonary manifestations of Sjögren's syndrome the presence of alveolar neutrophils was associated with a rapid decrease of DLco. Therefore, granulocytic alveolitis seems to lead to progressive pulmonary fibrosis irrespective of the underlying disease (SSc, Sjögren's syndrome or cryptogenic).

It is tempting to speculate that within the prognostically heterogenous group of patients with FASSc especially those with BALF granulocytosis should receive immunosuppressive treatment to slow the progression of pulmonary fibrosis, while patients with lymphocytic alveolitis may be followed up without such treatment. Randomised treatment studies are certainly needed to investigate this issue. Moreover, these investigations should also answer the question if treatment may convert a granulocytic (active, progressive) into a lymphocytic (inactive, non-progressive) alveolitis.

Taken together, the results of this study demonstrate that BALF differential cell count is a valuable prognostic parameter in FASSc. While normal BALF and BALF lymphocytosis are associated with a benign disease course, BALF granulocytosis predicts progressive FA with deterioration of lung function, which is most sensitively monitored by DLco. Thus, immunosuppressive therapy is recommended in patients with granulocytic FASSc.

1 Weaver AL, Divertie MB, Titus JL. Pulmonary scleroderma Dis Chest 1968;54:490-8

2 Rossi GA, Bitterman PB, Rennard SI, Ferrans VJ, Crystal RG. Evidence for chronic inflammation as a component of the interstitial lung disease associated with progressive systemic sclerosis. Am Rev Respir Dis 1985;124:612-17.

3 Harrison NK, Myers AR, Corrin B, Soosay G, Dewar A, Black CM, et al. Structural features of interstitial lung disease in systemic sclerosis. Am Rev Respir Dis 1991;144: 706-13.

4 Silver RM, Miller KS, Kinsella MB, Smith EA, Schabel SI. Evaluation and management of scleroderma lung disease using bronchoalveolar lavage. Am J Med 1990;88:470-6.

5 Wallaert B, Hatron PY, Grosbois JM, Tonnel AB, Devulde $\mathrm{B}$, Voisin C. Subclinical pulmonary involvement in connective tissue diseases assessed by bronchoalveolar lavage: relationship between alveolitis and subsequent changes in lung function. Am Rev Respir Dis 1986;133:574-80.

6 Rudd RM, Haslam PL, Turner-Warwick M. Cryptogenic fibrosing alveolitis - relationship of pulmonary physiology and bronchoalveolar lavage to response to treatment and prognosis. Am Rev Respir Dis 1982;124:1-9.

7 Wrognosis. Am Rev Respir Dis 1982;124:1-9. G. Diagnosis of alveolitis in interstitial lung manifestation in connective tissue diseases: importance of late inspiratory in connective tissue diseases: importance of late inspiratory
crackles, 67 gallium scan and bronchoalveolar lavage. Lupus 1996;5:606-12.

Wells AU, Hansell DM, Rubens MB, King AD, Cramer D Black CM, et al. Fibrosing alveolitis in systemic sclerosis. Arthritis Rheum 1997;40:1229-36.

9 Subcommittee for Scleroderma Criteria of the American Rheumatism Association Diagnostic and Therapeutic Criteria Committee: Preliminary criteria for the classification of systemic sclerosis (scleroderma). Arthritis Rheum 1980 23:581-90.

10 Miller NL, Miller RR. Computed tomography of chronic diffuse infiltrative lung disease. Am Rev Respir Dis $1990 ; 142: 1206-15$

11 Krause A, Hohberg B, Heine F, John M, Burmester GR, Witt C. Cytokines derived from alveolar macrophages induce fever after bronchoscopy and bronchoalveolar lavage. Am J Respir Crit Care Med 1997;155:1793-7. 
12 American Thoracic Society. Clinical role of bronchoalveolar lavage in adults with pulmonary disease. Am Rev Respir Dis 1990;142:481-6.

13 Salaffi F, Manganelli P, Carotti M, Baldelli S, Blasetti, Subiaco S, et al. A longitudinal study of pumonary involvement in primary Sjogren's syndrome: relationship between ment in primary Sjogren's syndrome. relationship between alveolitis and subsequent lung changes on high-resolution

14 Chan KL, Currie PJ, Seward JB, Hagler DJ, Mair DD, Tajik AJ. Comparison of three Doppler ultrasound methods in the prediction of pulmonary artery disease. J Am Coll Cardiol 1987;9:549-53.

15 Jacobsen S, Halberg P, Ullman S, Hoier-Madsen M, Petersen J, Mortensen J, et al. A longitudinal study of pulmonary function in Danish patients with systemic sclerosis. monary function in Danish patients

16 Behr J, Vogelmeier C, Beinert T, Meurer M, Krombach F, Konig G, et al. Bronchoalveolar lavage for evaluation and management of scleroderma disease of the lung. Am J Respir Crit Care Med 1996;154:400-6.

17 Bolster MB, Ludwicka A, Sutherland S, Strange C, Silver RM. Cytokine concentrations in bronchoalveolar lavage fluid of patients with systemic sclerosis. Arthritis Rheum 1997;40:743-51.

18 Wells AU, Hansell DM, Rubens MB, Cullinan P, Haslam PL, Black CM, et al. Fibrosing alveolitis in systemic sclerosis. Bronchoalveolar lavage findings in relation to computed tomographic appearance. Am J Respir Crit Care Med 1994;150:462-8

19 Ungerer RG, Tashkin DP, Furst D, et al. Prevalence and clinical correlates of pulmonary arterial hypertension in progressive systemic sclerosis. Am J Med 1983;75:65-74.

20 Salaffi F, Manganelli P, Carotti M, Baldelli S, Blasetti P, Subiaco S, et al. A longitudinal study of pulmonary involvement in primary Sjögren's syndrome: relationship between alveolitis and subsequent lung changes on high resolution computed tomography. Br J Rheumatol 1998;37:263-9. 